\title{
DISEÑO DE UN MODELO DE DIRECCIÓN POR COMPETENCIAS BÁSICAS DISTINTIVAS PARA LAS PYMES EXPORTADORAS DE LA CIUDAD DE MEDELLÍN ${ }^{*}$
}

\author{
Robert Ng Henao** \\ Abraham Londoño Pineda*** \\ Recibido: octubre 11 de 2010 • Aceptado: noviembre 13 de 2012
}

\section{RESUMEN}

El presente trabajo permite reconocer el modelo de dirección por competencias básicas distintivas (DCBD), como herramienta para que las pymes exportadoras de Medellín puedan medir su competitividad internacional, a partir de los recursos, competencias y capacidades existentes en su estructura administrativa y organizacional. Para ello se diseña un modelo de tipo cualitativo no probabilístico que combina escalas de medición de linkert con otras de diferencial semántico, cuyo producto es una matriz que permite clasificar la competencia esencial en tres categorías: valiosa, rara y exclusiva. De esta manera, las empresas pueden conocer qué tan preparadas se encuentran, en términos de sus recursos y capacidades, para internacionalizarse. El factor innovador de esta herramienta consiste en adicionar a los modelos de competencias básicas distintivas la orientación hacia la internacionalización.

\section{PALABRAS CLAVE}

Teoría de los recursos y capacidades, competencia esencial, competencias básicas distintivas, ventajas competitivas, internacionalización, pyme.

\section{CLASIFICACIÓN JEL}

M2

\section{CONTENIDO}

Introducción; 1. El modelo de gestión por competencias básicas distintivas dentro de los modelos de capital intelectual; 2. Perfiles basados en competencias básicas distintivas; 3. Diseño de la metodología; 4. Hallazgos; 5. Conclusiones; Bibliografía.

\footnotetext{
* Artículo de investigación es producto del proyecto "Diseño de un modelo sistémico de dirección por competencias básicas distintivas para las pymes exportadoras de la ciudad de Medellin", adscrito al Grupo de Investigación de Negocios Internacionales, el cual fue financiado por la Universidad de Medellín y ejecutado en el año 2010.

** Economista Industrial, Universidad de Medellín, Medellín, Colombia; Especialista en Finanzas Corporativas y Mercado de Capitales, Universidad Pontificia Bolivariana, Medellín, Colombia; Magister en Administración, Universidad de Medellín, Medellín, Colombia. Coordinador de la Maestría en Administración- MBA de la Universidad de Medellín, Colombia. Docentes del programa de Negocios Internacionales. Dirección Carrera 87 No 30-65, bloque 12, oficina 104, Universidad de Medellín, teléfono (57 4) 3405562 . Correo electrónico: robertng@udem.edu.co.

${ }^{* * *}$ Economista, Universidad Nacional de Colombia, Medellín, Colombia; Magister en Administración, Universidad de Medellín, Medellín, Colombia. Docente de tiempo completo del programa de Negocios Internacionales, Universidad de Medellín, Colombia. Coordinador de la UOC de Investigaciones del programa de Negocios Internacionales, Universidad de Medellín, Colombia. Dirección Carrera 87 No 30-65, bloque 12, oficina 104, Universidad de Medellín, teléfono (57 4) 340 5683. Correo electrónico: alondono@udem. edu.co.
} 


\section{DISTINCTIVE BASIC COMPETENCES MANAGEMENT MODEL DESIGN FOR EXPORTING SMES IN MEDELLIN CITY.}

\section{ABSTRACT}

This works allows to recognize the management model for basic distinctive competences (MBDC) as a tool for the exporting SMEs in Medellin for measuring their international competitiveness parting from the existent resources, competences and capacities inside their administrative and organizational structure. For this, a non probabilistic qualitative model is designed that combines the Linkert measuring scales with other with a semantic differential, whose product is a matrix for classifying essential competition in three categories: valuable, rare and exclusive. With this, companies can learn how well prepared they are in terms of their resources and capacities for their internationalization. The innovative factor of this tool is to add to the basic distinctive competence model an internationalization orientation.

\section{KEY WORDS}

Resource and capacity theory, essential competition, distinctive basic competences, competitive advantages, internationalization, SMEs.

\section{JEL CLASSIFICATION}

M2

\section{CONTENIDO}

Introducción; 1. El modelo de gestión por competencias básicas distintivas dentro de los modelos de capital intelectual; 2. Perfiles basados en competencias básicas distintivas; 2.1. Recursos; 2.2. Capacidades; 2.3. Rutinas; 2.4. Competencias básicas distintivas; 3. Diseño de la metodología; 4. Hallazgos; 5 . Conclusiones; Bibliografía.

\section{DISEHO DE UM MODELO DE DIREÇÃO POR COMPETENCIAS BÁSICAS DISTINTIVAS PARA AS PYME EXPORTADORAS DA CIDADE DE MEDELLIIN}

\section{RESUMO}

O presente trabalho permite reconhecer o modelo de direção por competências básicas distintivas (DCBD), como ferramenta para que as pyme exportadoras de Medellín possam medir sua competitividade internacional, a partir dos recursos, competências e capacidades existentes ao interior de sua estrutura administrativa e organizacional. Para isto se desenha um modelo de tipo qualitativo não probabilístico que combina escalas de medição de linkert com outras de diferencial semântico, que tem como produto uma matriz que permite classificar a competência essencial em três categorias: valiosa, raridade e exclusiva. Desta maneira, as empresas poderão conhecer que tão preparadas se encontram, em termos de seus recursos e capacidades, para se internacionalizar. O fator inovador desta ferramenta consiste em adicionar aos modelos de competências básicas distintivas a orientação há internacionalização.

\section{PALAVRAS CHAVES}

Teoria dos recursos e capacidades, competência essencial, competências básicas distintivas, vantagens competitivas, internacionalização, pyme.

\section{CLASIFICAÇÃO JEL}

M2

\section{CONTEUDO}

Introdução; 1 . O modelo de gestão por competências básicas distintivas dentro dos modelos de capital intelectual; 2. Perfis baseados em competências básicas distintivas; 2.1. Recursos; 2.2. Capacidades; 2.3. Rotinas; 2.4. Competências básicas distintivas; 3 . Desenho da metodologia; 4. Descobertas; 5 . Conclusões; Bibliografia. 
Diseño de un modelo de dirección por competencias básicas distintivas para las PYMES exportadoras de la ciudad de Medellín

\section{INTRODUCCIÓN}

Debido a la rapidez y dinamismo con el que gira el mundo en lo referente a los aspectos económico y empresarial, las organizaciones están llamadas a afrontar con mayor premura la decisión de actuar en el ámbito internacional. Por lo tanto, ante las dificultades que se movilizan en el interior de las empresas en la disposición de los recursos, capacidades y conocimientos se hace necesaria la medición de los mismos como condición previa para un planeado proceso de internacionalización.

Para Lado y Wilson (1994, p.711), el aprendizaje organizacional ha sido considerados como una competencia básica distintiva, que se constituye en un fuerte potencial para el desarrollo de ventajas de carácter comparativo, competitivo y sostenido en las organizaciones empresariales, aunque, al establecer el impacto del aprendizaje en los resultados de las estructuras productivas y su potencial de internacionalización, es muy poco lo que se conoce.

Al respecto, los trabajos de Fiol y Lyles, (1985, p.809); Dogson, Bontis, Crossan y Hulland, (2002, p. 444) hacen una defensa de la correspondencia entre la identificación de competencias y la mejora en los resultados obtenidos por la empresa pero no profundizan en la temática de la competitividad internacional. Incluso, en las aportaciones de Crossan, Lane y White, (1999, p. 527) se pone de manifiesto que, ni siquiera, esa primera relación podría ser tan directa.

Cardona y Calderón (2006, p.13), al abordar los trabajos de Tsang (1997) plantean que "aún no son claras las características y las condiciones desde las cuales el aprendizaje e identificación de competencias básicas, como proceso generador de conocimiento, tiene un impacto positivo en los resultados del negocio y mucho más en los procesos de internacionalización".

Por este motivo, la evidencia en materia de estudios que respalden la relación entre la identificación de competencias básicas como herramientas para medir la competitividad y la gestión internacional de las empresas es poca. Malaver y Vargas (2004) encuentran que solo en el 29\% de los casos, las estrategias competitivas de las empresas se orientan a generar capacidades distintivas como el aprendizaje. Otros trabajos, como el de Cardona y Calderón (2006, p. 14-16), centran su objeto de estudio en la relación entre el aprendizaje organizacional y el desempeño empresarial, en el que se supone que aquel es una competencia básica distintiva que puede ser explicada desde la teoría de recursos y capacidades, y este un constructo social que se mide a través de la percepción de su rendimiento. Cardona y Calderón (2006, p. 12) explican que:

El contraste empírico se efectuó en 45 empresas medianas y grandes, con un diseño cuantitativo de tipo explicativo, para lo cual se emplearon métodos

Semestre Económico, volumen 15, №. 32, pp. 197-224 • ISSN 0120-6346, julio-diciembre de 2012, Medellín, Colombia 
Robert Ng Henao • Abraham Londoño Pineda

multivariados como los análisis de varianza (Anova), de clústeres y de componentes principales, cuyos resultados arrojaron evidencia que permite aceptar la hipótesis de que la capacidad de aprendizaje influye en el desempeño de la organización.

De igual forma, Calderón (2006, p. 57) realizó un estudio en el que relacionó la actividad internacional de las pequeñas y medianas empresas con sus competencias básicas distintivas; él se centró en las competencia de tipo organizacional, en especial, en la variable que denominó competitividad empresarial. Este estudio se aplicó en 16 unidades productivas de la ciudad de Manizales; para ello utilizó un enfoque cualitativo cuya orientación fue de tipo estratégico y de gestión humana. Sin embargo, por las características de este no se pudo generar una representación de tipo estadístico que validara dicha relación.

Lo hasta acá presentado hace evidente el vacío en términos de desarrollos metodológicos orientados a evaluar la competitividad internacional, a partir de los recursos, competencias y capacidades existentes en la estructura administrativa y organizacional de las empresas. Por esta razón, el trabajo que se presenta a continuación tiene como objetivo el diseño de una metodología que permita medir el potencial de internacionalización a través de las competencias básicas distintivas que poseen las organizaciones, dado que en el actual contexto de internacionalización y globalización de las estructuras económicas, uno de los propósitos de toda empresa, sobre todo, si está catalogada dentro de la clasificación de pequeña y mediana empresa, es el de fijar límites organizacionales, más allá de las fronteras nacionales, a partir de los recursos, capacidades y competencias de las cuales disponga, sin que se llegue a notar la transnacionalización de su esencia productiva y competitiva.

En este contexto, la importancia de los posibles aportes de trabajos como este se circunscribe con la línea número 3 del Plan de Desarrollo de la ciudad Medellín 2007-2010 (Alcaldía de Medellín, 2008, p. 2-3), en lo referente a su primer componente de creación y fortalecimiento de empresas, cuyo propósito es la promoción de estructuras productivas innovadoras y sostenibles en la ciudad, orientadas a mejorar el desarrollo del tejido empresarial a través de programas tales como apoyo al emprendimiento, emprendimiento barrial, emprendimiento social y rural, fortalecimiento de MIPYME, apoyo a comunidades clúster y fortalecimiento de la productividad.

Este diseño metodológico se desarrolla bajo la óptica de una perspectiva cualitativa en la que se identifica una serie de conceptos principales (constructos) y secundarios (subconstructos), se definen unas variables dependientes (competencia esencial) e independientes (competencias básicas distintivas), se establece una 
Diseño de un modelo de dirección por competencias básicas distintivas para las PYMES exportadoras de la ciudad de Medellín

serie de supuestos (constantes y parámetros), se toma un número de elementos que componen las competencias básicas distintivas (ítems), los que sirven como guía para orientar las preguntas que se hacen en cada competencia distintiva, y se establecen unas escalas de medición que, para este caso, son de tipo Linkert ${ }^{1}$ combinadas con otras de diferencial semántico² que dan la posibilidad de cuatro opciones de respuesta (1,2 3 y 4); a medida que se elija una respuesta de más alto valor numérico significa que la empresa tiene un mayor compromiso de sus recursos y capacidades con el propósito de internacionalización. Ello da como resultado una matriz que permite vincular las competencias de las empresas con su potencial de internacionalización y se presenta como un instrumento útil para gestionar los recursos y las capacidades de estas en pro de la consolidación de procesos de inserción internacional.

El principal aporte de este trabajo consiste en el hecho de generar un valor incremental a la clasificación tradicional de la competencia esencial: valiosa, rara y exclusiva, desarrollada en los trabajos de Bueno y Morcillo (1997), al vincular a su medición, el potencial de internacionalización. De esta manera, las empresas que apliquen este instrumento podrán a través de una medición saber qué tan preparadas se encuentran para internacionalizarse; en la medida en la que su clasificación avance hacia la categoría exclusiva querrá decir que se encuentran más preparadas en términos de sus recursos y capacidades para operar en mercados foráneos.

Adicional a lo anterior, esta herramienta permite identificar recursos y capacidades estratégicas que deben ser llevados a un plan de mantenimiento, y otros, débiles, que tienen que ser intervenidos por medio de planes de mejoramiento. Es decir, que sirve, además, como instrumento para la gestión de los recursos y las capacidades de las organizaciones.

La estructura del presente trabajo concentra, en un primer momento, la ubicación del modelo de competencias básicas distintivas a la luz de los diferentes modelos de capital intelectual, se describe la evolución de los diferentes enfoques de analisis y su importancia en los procesos de gestión organizacional.

1 Es una escala psicométrica comúnmente utilizada en cuestionarios, y es la escala de uso más amplio en encuestas para la investigación, principalmente en ciencias sociales. Al responder a una pregunta de un cuestionario elaborado con la técnica de Likert, se especifica el nivel de acuerdo o desacuerdo con una declaración (elemento, ítem o reactivo o pregunta). La escala se llama así por Rensis Likert, quien publicó en 1932 un informe donde describía su uso.

2 Es un instrumento de evaluación psicológica creado por Charles Osgood, George Suci y Percy Tannenbaum en 1957. Este instrumento se sustenta en la teoría mediacional de dichos autores, de corte neoconductista. Se plantea que un concepto adquiere significado cuando un signo (palabra) puede provocar la respuesta que está asociada al objeto que representa; es decir, se reacciona ante el objeto simbolizado.

Semestre Económico, volumen 15, №. 32, pp. 197-224 • ISSN 0120-6346, julio-diciembre de 2012, Medellín, Colombia 
En una segunda parte se presentan los perfiles basados en competencias básicas distintivas, se hace énfasis en la ilustración de los conceptos de recursos, capacidades, rutinas y competencias básicas distintivas.

Un tercer momento muestra el diseño metodológico a través de la estructura que soporta la construcción del modelo: definición de constructos, subconstructos, variables, constantes, parámetros, ítems, medidas y escalas de medición.

Este diseño metodológico se presenta como un aporte incremental a los modelos de competencias básicas distintivas desarrollados por Sáez de Viteri (2000) y Bueno y Morcillo (1997). Lo novedoso de este modelo radica en dos asuntos:

Primero, en la cuantificación de las distintas competencias; para ello se usan unas escalas de medición de Linkert combinadas con unas de diferencial semántico utilizadas para dar cuatro tipos de respuestas. En la medida en que las respuestas dadas por las empresas tienen un valor numérico más alto, las empresas presentan un mayor compromiso de sus recursos y capacidades con respecto a su propósito de internacionalización.

Segundo, en la orientación hacia la internacionalización; ello se debe a que las preguntas que conforman los ítems propios de cada competencia distintiva se enfocan a la temática de la internacionalización de las empresas; los dos modelos anteriores eran genéricos.

\section{EL MODELO DE GESTIÓN DE COMPETENCIAS BÁSICAS DISTINTIVAS DENTRO DE LOS MODELOS DE CAPITAL INTELECTUAL}

Al inicio de la década de los noventa, la puesta en acción de la actividades de I+D generó un aumento del valor de mercado de las organizaciones, por encima del valor contable reflejado en los libros y balances de las compañías; esa diferencia consistía en que aquellas utilizaban un capital adicional que generaba valor y que otras organizaciones no lo valoraban; se trataba, entonces, de capital intelectual o intangible. De inmediato, revistas especializadas como Forbes, Industry Week, Angeles Time y Fortune comenzaron a difundir información y a crear conocimiento sobre el tema (Bueno, 2005, p.2).

El primer enfoque dado al capital intelectual se conoció con el nombre de perspectiva financiero-administrativa; entre sus autores más reconocidos, se ubican los aportes de Skandia (1992); Edvinson (1997); Brooking (1996); Bontis (1996); Sveiby (1997). Esta visión se centró en una visión contable que, solo identificó los asuntos generadores de valor en aspectos como el servicio al cliente, los recursos humanos de la organización y la renovación de los procesos; para este las organizaciones que 
Diseño de un modelo de dirección por competencias básicas distintivas para las PYMES exportadoras de la ciudad de Medellín

se diferencian en tales asuntos son las que pueden marcar diferencia en el mercado y generar un valor por encima del estimado en sus cuentas de balance.

Con el paso del tiempo el concepto evolucionó bajo la armonización de los capitales; a este enfoque se le dio el nombre de estratégico-corporativo. En él, los conceptos de capital humano, tecnológico, organizativo y relacional empezaron a usarse de manera recurrente y organizada. En esta perspectiva se reconocen los modelos de Atkinson y otros (1997); Bontis (1998) y Bueno (1998).

Posterior a ello se desarrolla el enfoque social-evolutivo, que abarca los modelos presentados por Camison, Palacios y Devece (2000) denominado American Society for Training and Development, y el desarrollado por McElroy (2001) conocido como modelo Intellectus En este tipo de modelos se presenta ya una madurez del concepto y se exhibe una combinación de componentes o capitales de una manera más compleja y dinámica. Tales capitales son: el social, cultural, de innovación o de emprendimiento, entre otros.

Los desafíos que en la actualidad enfrentan las organizaciones, en materia de productividad y competitividad ofrecen un reto puntual para la economía y la administración; este reto lo constituye el manejo eficiente del conocimiento. El conocimiento y la información representan hoy el núcleo básico de cualquier organización, de forma que ninguna puede existir sin una gestión eficiente, válida y fiable que contribuya a centrar de forma estratégica sus decisiones y operaciones.

Es importante señalar que el contexto actual de las organizaciones, tal y como lo manifiesta Castells (1995, p. 11) al tratar de describir a la sociedad como una sociedad de la información, parte de un nuevo sistema tecnológico, económico y social, inmerso en uno donde el crecimiento de la productividad no depende del incremento cuantitativo de los factores de producción (capital, trabajo y recursos naturales), sino de la incorporación de conocimientos e información a los procesos de gestión, dirección producción y distribución de cualquier estructura productiva en particular.

Esta evolución fue bien entendida por varios autores pioneros de la revolución del conocimiento en el sistema productivo tradicional, entre ellos Sakaya (1985), en donde dimensionó hace 20 años lo que aún hoy pocos alcanzan a ver, lo cual describe de la siguiente forma (Sakaya, 1985):

El poder económico de una nación es la resultante de todos los elementos de valor que se expresan en una sociedad; que para fundar el desarrollo sostenible de una ciudad o región se requiere tanto capital racional como emocional, financiero como relacional, tecnológico como cultural; que no es 
la acumulación de capital (crecimiento) sino el balance del mismo (el desarrollo integral y sustentable) lo que permite resultados en identidad, salud, cohesión y viabilidad futura a una sociedad mucho más equitativa y lo que constituye, por tanto, la verdadera riqueza de las naciones.

El sistema de dirección básica por competencias distintivas es un modelo de capital intelectual ubicado en el enfoque estratégico-corporativo, de forma más específica en el modelo de Bueno y Morcillo (1997). Este se presenta como un paradigma de la dirección estratégica que busca identificar la fuente real de la ventaja competitiva sostenida, bajo un enfoque de eficiencia y productividad en el interior de las organizaciones. Esta corriente de pensamiento se sustenta en criterios como los que exhiben: Simon (1957) en su enfoque de racionalidad limitada, Wernelfelt (1984) en sus conceptualizaciones acerca de la heterogeneidad empresarial, Nelson y Winter (1982) en sus trabajos sobre la dependencia histórica, y Barney (1986) en sus aportes al tema de la imperfección del mercado de factores estratégicos. Para éste último, los conceptos centrales son los recursos, las rutinas y las capacidades propias de las organizaciones.

En el modelo de dirección de Bueno y Morcillo (1997), la competencia esencial está compuesta por tres habilidades distintivas: una de origen estructural (capital tecnológico y organizativo), otra del ámbito o del entorno socio económico (capital relacional) y una de carácter personal (capital humano). De estas tres se obtiene la Core competencies o competencia esencial, que es la que genera que una organización sea diferente a otra y que consolide una ventaja competitiva propia y sostenida.

Además, el modelo de dirección por competencias básicas delinea tres argumentos primordiales; el primero se encuentra relacionado con las actitudes o valores personales, tecnológicos, organizativos y relacionales, los que representan en definitiva lo que quiere hacer la empresa.

El segundo trata de las aptitudes y recursos incorporados en las competencias distintivas, es decir, que en cuanto al capital humano significan aptitudes personales; con respecto a la tecnología, se refiere a los recursos tecnológicos, y en lo concerniente a lo organizativo, indica lo relacionado con el aprendizaje organizacional; dichas aptitudes en definitiva representan lo que hace o sabe hacer la empresa.

El tercer argumento es el de las capacidades; si se habla de las que se encuentran relacionadas con el capital humano, entonces, se hace alusión a las habilidades y experiencia del personal de la organización; si se cruza con el capital estructural, pero de forma más específica con el componente tecnológico, entonces, se aduce a las capacidades tecnológicas y de innovación en procesos y productos, y al mencionar las capacidades organizativas, se hace necesario profundizar en las habilidades 
Diseño de un modelo de dirección por competencias básicas distintivas para las PYMES exportadoras de la ciudad de Medellín

directivas y de liderazgo del equipo de gestión, con lo que se tendrá en síntesis lo que es capaz de hacer y de ser la empresa mejor que la competencia, lo que será difícil de imitar y que se constituye en la fuente de su ventaja competitiva.

El correcto diseño e implementación de un modelo basado en competencias debe poseer la capacidad de aportar unas bases sólidas para la administración eficiente del recurso humano, además de poseer la capacidad de alinear y apoyar las necesidades del negocio. Un modelo de esta clase es desarrollado con contribuciones extensas de los líderes del negocio y operaciones; apalanca las capacidades analíticas, encontradas dentro de las funciones de recursos humanos y sirve como una plataforma común y consistente para una variedad de iniciativas adicionales a través de las cuales, la gestión de lo humano puede hacer avanzar los intereses estratégicos de negocio.

Cabe resaltar que se ha producido una ruptura del método denominado taylorista, en el que el trabajador tenía un puesto y una función prevista de antemano y, por tanto, muy rígida, para ir a una nueva escala de valores más flexible en la que se valora al capital humano como un conjunto de actitudes, habilidades y conocimientos puestos a disposición de un colectivo empresarial.

Las empresas se conciben hoy como un conjunto de personas que llevan a cabo actividades para la consecución de objetivos planificados. El éxito o fracaso de la empresa reside en el conocimiento o aprovechamiento de las capacidades, habilidades, conocimientos y actitudes de las personas para la consecución de los objetivos que se ha fijado. Hoy triunfan las empresas que saben desarrollar su potencial, su personal, los métodos y sistemas. Por esto, la tendencia de las empresas se dirige hacia la gestión del talento humano y la humanización de la gestión, al potenciar las características del personal en función de los roles, puestos y tareas.

Uno de los referentes que reafirma la idea general, a la que se pretende llegar con el diseño de un modelo de competencias basado en las competencias básicas distintivas, fue tratado por Calderón (2006, p. 59), quien afirma lo siguiente:

La importancia de las personas en el proceso de aprendizaje organizacional y en la generación de competencias básicas distintivas ha sido constatada por diversos investigadores. Pfeffer (1996 y 1998) y Ulrich (1997) han estudiado la importancia de las personas en generar ventaja competitiva sostenida. Así, por ejemplo, comprometer al trabajador con la empresa mediante políticas como el pago contingente aumenta la predisposición a adquirir nuevos conocimientos y habilidades e incentiva la existencia de iniciativa por parte de los trabajadores; Cuervo (1993) reconoce que la dimensión humana se ha convertido en factor clave de la productividad por cuanto ésta depende, en buena medida, del

Semestre Económico, volumen 15, №. 32, pp. 197-224 • ISSN 0120-6346, julio-diciembre de 2012, Medellín, Colombia 
desarrollo de competencias de las personas, incluso más que de la inversión en capital (específicamente afirma que el crecimiento vía cambio tecnológico depende en mayor proporción de la inversión en educación que de la inversión en equipos). En este mismo sentido, Huselid (1995) encontró en su estudio que determinadas prácticas de gestión humana tienen impactos positivos sobre la productividad empresarial, v. gr., el desarrollo de políticas de retención de trabajadores cualificados.

Sin embargo, es importante aclarar que en el diseño de un modelo de competencias básicas distintivas, la cuantificación del valor de la competencia esencial puede variar debido a dos factores: el primero es la forma como se hagan las preguntas orientadas a la obtención de información alrededor de las competencias básicas distintivas (estratégicas, tecnológicas, organizacionales y de personal) y su orientación hacia la internacionalización; el segundo tiene que ver con las opciones de respuesta y su grado de profundidad. Sin embargo, el rigor con el que se ha diseñado el modelo permite que este sea aplicado como una alternativa para conocer el nivel de competencia esencial en el que se encuentra cada organización.

Los resultados de la competencia esencial les permiten a las empresas conocer su posición competitiva y las brechas que deben cerrar para avanzar hacia una competencia de tipo exclusivo. A partir de ello se debe, entonces, redefinir la estrategia competitiva, ahora con una visión más amplia, para incursionar o para afianzarse en los mercados internacionales, según sea el caso.

\section{PERFILES BASADOS EN COMPETENCIAS BÁSICAS DISTINTIVAS -CBD-}

En Colombia, por cerca de 60 años, predominó un modelo de economía cerrada, basado en la sustitución de importaciones que llevó de manera progresiva a promocionar las exportaciones, pero también ocasionó que las decisiones empresariales se enfocaran de manera estricta hacia el mercado local, al generar toda serie de atrasos tecnológicos y poca capacidad para la reconversión industrial. Ante el avance del fenómeno de la globalización, se hace más difícil permanecer y competir en los mercados locales y en lo foráneos, por lo que es necesario que las empresas se diferencien de los competidores, al ofrecer productos que según Barney (1991) deben ser valiosos, raros, inimitables o insustituibles y con alto valor agregado.

Para Navas y Guerra (1998), el modelo de dirección por competencias básicas distintivas se enmarca dentro del llamado análisis estratégico de la organización, al hacer parte de los enfoques que pertenecen al punto de vista interno de la organización, lo que significa que debe ser complementado con el analisis externo (contexto económico, social,político, tecnolólgico y cultural que la rodea y la competencia). 
Diseño de un modelo de dirección por competencias básicas distintivas para las PYMES exportadoras de la ciudad de Medellín

Según Sáez de Viteri (2000, p. 73) las empresas deben buscar potenciar sus generadores de valor; es decir, fortalecer los activos estratégicos, al entender por estos sus recursos, capacidades y rutinas.

\subsection{Los recursos}

Penrose (1959, p.27) define a la empresa como una colección de recursos productivos, que pueden ser físicos como: planta, equipo, recursos naturales, materia prima; o intangibles: legales como marcas, patentes; u organizacionales como la cultura corporativa.

Uribe (2001) los clasifica como tangibles e intagibles y estos últimos son de dos formas: formales (patentes, licencias, contratos) e informales (conocimientos en el ámbito organizacional, individual y conocimientos y habilidades del equipo de gestión). Estos últimos fueron catalogados por Winter (1987) como de dificil apropiación, y se configuran como los activos estratégicos de las organizaciones y su fuente de ventaja competitiva.

Aunque Barney (1991, p. 102) considera que no todos los activos que posee una empresa pueden ser considerados como recursos, solo aquellos que exploten las oportunidades y neutralicen las amenazas del entorno se podrán considerar como atributos válidos y por tanto recursos generadores de ventaja competitiva.

\subsection{Las capacidades}

Para Uribe (2001), no es suficiente poseer numerosos recursos sino, más aún, la manera en que estos se gestionan, así como las posibilidades reales de transferencia, su recepción y explotación. Este trabajo depende, en gran parte, de la alta dirección; es decir, del liderazgo que tenga el equipo de gestión de la organización debido a que su labor se asocia a la búsqueda, incorporación, renovación y sostenimiento de las capacidades de la empresa.

Amit y Schoemaker (1993, p. 36) hicieron un gran aporte al integrar las teorías de los recursos con aquellas que hablan de las capacidades. Los recursos representan el stock o acumulado de factores disponibles que controlan las empresas y su interrelación forma el conjunto de capacidades de la empresa. En las primeras teorías, las de los recursos, la ventaja competitiva viene dada por los recursos que poseen las organizaciones, mientras en el segundo enfoque, el de las capacidades, importa mucho más la forma como se gestionen y utilicen los mismos.

En general, lo que se trata de establecer es que los recursos por sí solos no proveen a la empresa de una ventaja competitiva, sino que esta depende de la manera como se utilizan y con qué habilidad se gestionan. 


\subsection{Las rutinas}

Según Nelson y Winter (1982, p.73) una rutina es lo que mejor sabe hacer la organización. Este autor plantea que las empresas saben cómo hacer las cosas porque disponen de unas rutinas apropiadas. Cuando la empresa define lo que hay que hacer y el cómo hacerlo da a conocer las rutinas.

El proceso de aprendizaje organizacional se sustenta en el despliegue, repetición, imitación o emulación de rutinas y tareas que conllevan a una más eficiente y rápida ejecución de las diferentes funciones. Una organización no presenta alteraciones continuas en su estructura organizacional porque ello implicaría la implementación de cambios recurrentes en las rutinas, lo que se podría convertir en un actividad destructora de valor y en un obstáculo para consolidar y mantener la ventaja competitiva.

Podría darse el caso en el que la diferencia entre dos organizaciones no fueran los recursos, ni las capacidades, sino las rutinas. Puede ser que la forma como se hacen las cosas en una organización sea la fuente de ventaja competitiva y que dichas rutinas sean difíciles de copiar por los competidores.

Con este trabajo, también, se pretende mostrar que los recursos, capacidades y rutinas tienen cada vez un papel más relevante en el mundo actual y en la estrategia de las organizaciones. Por ello, es importante mirar el beneficio de las empresas en función de las características del entorno y de los recursos, capacidades y rutinas de que disponen.

\subsection{Las competencias distintivas}

El concepto más importante del presente trabajo es el de competencias distintivas. Por ello, es importante hablar acerca de ellas.

Prahalad y Hamel (1990) le dieron el nombre de nucleares, y las entendieron como las habilidades que contribuyen a aumentar el valor agregado, que son percibidas por los clientes y son tan exclusivas de las empresas que permite distanciarlas de los competidores. Además, plantean que las organizaciones deben identificar y mejorar las competencias sobre las que fundamentan su ventaja competitiva de largo plazo.

Por su parte, Quin y Hilmer (1994), las llamaron competencias clave, y las asociaron a los conocimientos y destrezas, mas no a las funciones, ni tareas. Esta perspectiva permite dar un gran paso, ya que desde allí se considera no un enfoque basado en las actividades y tareas, sino en los procesos. 
Diseño de un modelo de dirección por competencias básicas distintivas para las PYMES exportadoras de la ciudad de Medellín

Hoy prefiere hablarse de competencias distintivas. Al respecto, Selznick (1957) fue el primero en mencionarlas. De igual forma, La AECA (1999) ${ }^{3}$, asemeja a las competencias distintivas como concepto equivalente a los de competencia nuclear y competencia clave. El aporte de esta asociación consistió en difundir una clasificación de las competencias distintivas en estratégicas, tecnológicas, personales y organizativas, las que articuladas con los recursos y capacidades se constituyen en los generadores de valor propios del análisis interno de la organización (Sáez de Viteri, 2000, p.75).

A continuación se presenta una aproximación a la definición que Arango y Gil (2008, p.120 - 123) hacen de las competencias básicas distintivas presentadas en los modelos de Bueno y Morcillo (1997):

La competencia estratégica: da la idea de la existencia de un proyecto orientado hacia los diferentes grupos de interés de la organización. Además, se asocia a la visión, la misión y los objetivos (lo que quiere ser la empresa), los recursos (lo que es y sabe hacer), las capacidades (lo que es capaz de hacer) y la gestión y coordinación de los anteriores.

La competencia tecnológica: expresa la capacidad de diseñar y fabricar que tiene la empresa. Los componentes serían: el stock de tecnologías, el saber cómo aplicarlas y su capacidad de innovación.

La competencia de personal: denota el conjunto de capacidades de las personas tácitas o explicitas. Es el llamado Etos de la organización que está compuesto por aptitudes o conocimientos (lo que saben hacer), habilidades (lo que pueden hacer) y las actitudes o comportamientos (lo que quieren hacer).

La competencia organizativa: se refiere a la coordinación de las capacidades, recursos y rutinas a través de la estructura organizativa de la empresa. Se encuentra conformada por el diseño de las tareas, rutinas y métodos, estructuras de poder, sistemas de dirección e información.

Lo que se pretende, entonces, es que mediante el analisis interno de la organización (recursos, capacidades y rutinas) y en conjunto con el analisis del entorno, las organizaciones logren identificar las competencias básicas distintivas para determinar, así, su competencia esencial, que permita a las empresas pymes construir una nueva estrategia, a partir de la que surje una nueva estructura para que la inserción de estas en los mercados internacionales se pueda dar de una mejor forma.

3 Asociación Española de Contabilidad y Administración 


\section{DISEÑO METODOLÓGICO}

Este trabajo presenta el diseño de un modelo de DCBD desde los desarrollos teóricos propuestos por Bueno y Morcillo (1997) y Sáez de Viteri (2000), cuya finalidad es medir el potencial de internacionalización por medio de la evaluación de las competencias básicas distintivas de las organizaciones: competencias estratégicas, tecnológicas, de personal y organizacionales, lo que permite no solo concentrar la gestión en ellas, sino identificar la competencia esencial y los activos estratégicos que sirven como fuente de ventaja competitiva para estas. Adicional a ello se busca que las empresas conozcan, posean y desarrollen recursos y capacidades adecuadas a las exigencias específicas del entorno en que la organización proyecta operar, en este caso, un escenario internacional.

\section{Constructos del modelo}

Para Moutinho (2011, p. 5) un constructo es un término o concepto teórico principal que es involucrado en el problema u objeto de investigación. En este trabajo los constructos principales son: las competencias básicas distintivas y la competencia esencial.

Sáez de Viteri (2000, p. 73) define la competencia esencial como aquello que permite diferenciarse de la competencia. Por esta razón la considera como fuente esencial de ventaja competitiva.

Por su parte, Uribe (2001, p. 28) especifica que la competencia esencial y las competencias básicas son un paradigma de la dirección estratégica perteneciente al análisis interno de las organizaciones que pretende identificar la ventaja competitiva de las mismas bajo un enfoque de eficiencia. Según este autor, la competencia esencial puede ser de tres tipos: la primera de ellas se da cuando el recurso estratégico es valioso. Este se define como aquel que permite explotar y neutralizar amenazas presentes en el entorno de la empresa; el segundo tipo de competencia esencial es la que se sustenta en un activo raro. Según Uribe (2001), "un activo es raro, en el sentido de que no lo posean otras empresas ya que ello le pondría límites a sus competidores"; la tercera forma en la que se presenta la competencia esencial es cuando la organización posee activos exclusivos. Estos, a su vez, pueden ser imitables o insustituibles.

Algo similar dejan ver en los trabajo de Sáez de Viteri (2000, p. 78):

Los recursos valiosos y raros solamente conservarán la posibilidad de ser exclusivos si las empresas que no los poseen no pueden conseguirlos porque son inimitables y no existen en el mercado para su negociación de transacción, ya que han sido conformados en el ámbito interno de la organización y ser, por lo tanto, únicos. 
Diseño de un modelo de dirección por competencias básicas distintivas para las PYMES exportadoras de la ciudad de Medellín

Por este motivo, la idea básica sería la de asociar el grado de calificación de la competencia esencial con el nivel de recursos y capacidades que la empresa tiene para hacer frente al proceso de internacionalización. Cuando esta salga evaluada en la categoría exclusiva, mejor preparada estará para afrontar dicho propósito.

\section{Subconstructos del modelo}

Según Moutinho (2011), los subconstructos son "conceptos derivados de los constructos principales que son relevantes en la investigación". Para este trabajo se tomaron los conceptos de recursos, capacidades y rutinas.

Para Penrose (1959) una empresa es

... una colección de recursos productivos, que pueden ser físicos como: planta, equipo, recursos naturales, materia prima, bienes semi-terminados; o intangibles: legales (marcas, patentes), organizacionales (cultura corporativa) y relacionales (reputación, relaciones con proveedores), y humanos (habilidad y conocimiento de la mano de obra calificada o no calificada, personal de oficinas administrativas, financiero, legal, técnico y directivo).

En sentido, Sáez de Viteri (2000) hace una precisión, al resaltar que "los factores esenciales en el proceso de producción no son los recursos en sí, sino los servicios que tales recursos rinden".

Uribe (2001, p. 30), por su parte, los clasifica como tangibles e intagibles y, a estos últimos, a su vez, en formales (patentes, licencias, contratos) e informales (conocimientos en el ámbito organizacional, individual y conocimientos y habilidades del equipo de gestión).

En la misma vía, Carrión (2007) destaca que "los recursos / activos relevantes son los de tipo intangible, debido a que aportan verdadero valor a las organizaciones, definiéndolos como una serie de recursos que pertenecen a la organización, pero que no están valorados desde un punto de vista contable".

Barney (1991), en cambio, plantea que los recursos tienen una relación con la ventaja competitiva que las empresas logran consolidar ya que ella es producto de los recursos que estas controlan. De esta manera, la teoría de los recursos propone que solo puede ser considerado como tal aquello que permita aprovechar las oportunidades y neutralizar las amenazas del entorno.

En este sentido Uribe (2001) resalta la necesidad de articular el concepto de recursos con el de capacidades ya que lo importante no son solo los recursos sino la manera en que estos se gestionan y sus posibilidades reales de explotación. 
Carrión (2007) da una aproximación a las capacidades. Para él una capacidad organizativa es la habilidad de una empresa que permite llevar a cabo una actividad concreta (en grupo). Por ello, los recursos deben ser bien gestionados si se quiere generar una capacidad. Esta habilidad depende, en gran parte, de la alta dirección; es decir, del liderazgo que tenga el equipo de gestión de la organización debido a que su labor se asocia a la búsqueda, incorporación, renovación y sostenimiento de las capacidades de la empresa.

Otros autores que aportan a la definición de capacidades son Ulrich y Wieserma (1989, p. 114). Para estos, las capacidades son la habilidad de una empresa para establecer estructuras y procesos internos con el objeto de crear competencias específicas que les permitan adaptarse a las cambiantes presiones externas, aunque las capacidades organizativas se centran en el ámbito interno de la empresa, en decisiones y prácticas dentro de la misma que modifican la forma en que esta responde a las presiones externas.

En general, lo que se trata de establecer es que los recursos por sí solos no proveen a la empresa de una ventaja competitiva, sino que esta depende de cómo se utilizan, y con qué habilidad se gestionan, o como lo presentan Amit E Schoemaker (1993, p.41), de la destreza o habilidad para combinar los recursos tangibles e intangibles con los que cuenta la empresa es como sobrevienen las capacidades o competencias de la misma.

Para el propósito de este trabajo, vale decir que para internacionalizarse no basta con poseer los recursos para hacerlo, sino que se requiere saber qué hacer con ellos y cómo gestionarlos de forma adecuada. Así, la combinación de la visión de la empresa, sus recursos y sus capacidades no solo serán los elementos generadores de la competencia esencial y, por lo tanto, de la ventaja competitiva de la organización sino que representan la base para un exitoso proceso de internacionalización.

\section{Variables del modelo}

El presente trabajo se hace bajo una perspectiva que concibe a las organizaciones como sistemas integrados por elementos que interactúan de forma continua y cuyas características y comportamiento están articulados para lograr objetivos concretos. Antes de definir las variables es importante recordar lo que se entiende por modelo según Moutinho (2011, p.5):

Un modelo es un conjunto de variables y sus interrelaciones diseñado para representar un sistema real o proceso, pero que no representa plenamente a esa misma realidad. 
Diseño de un modelo de dirección por competencias básicas distintivas para las PYMES exportadoras de la ciudad de Medellín

El objetivo de los modelos es proporcionar un sistema de predicción que pueda ser manipulado para estudiar la situación del mundo real con el fin de ayudar a quien toma las decisiones. Además, el modelo puede ser considerado para representar las relaciones entre las variables, constantes, y parámetros del sistema y su entorno.

Para el caso específico de este modelo, es necesario hacer referencia a unas variables de entrada (inputs), a partir de las cuales este proporciona un resultado final u output. Las variables son definidas desde los constructos principales, esto es, las competencias básicas distintivas y la competencia esencial.

\section{Variables inputs y output del modelo}

En este modelo las variables de entrada, también conocidas como inputs o variables independientes serán las competencias básicas distintivas. En el modelo de Bueno y Morcillo (1997) estas son de tipo estratégico, tecnológico, de personal y organizativo.

La variable de salida u output será la llamada competencia esencial. Esta variable operará como dependiente de las competencias distintivas, es decir, que lo que ocurra con las competencias estratégicas, tecnológicas, de personal y organizativas incidirá en el resultado de la competencia esencial.

Para Bueno y Morcillo (1997), la competencia esencial depende de la suma de las competencias básicas distintivas:

Competencia esencial $=$ competencia estratégica + competencia tecnológica + competencia de personal + competencia organizativa.

Debe recordarse que las valoraciones y criterios de calificación que se den acerca del estado de sus competencias básicas distintivas influyen sobre el resultado de su competencia esencial. De esta manera pueden dar tres tipos de competencia esencial: valiosa, se encuentra en autores como Barney (1991) y Porter (1991); rara y exclusiva, que es desarrollada por Barney (1991), Amit y Shoemaker (1993) y Teece et al (1994).

\section{Constantes y parámetros de medición}

Moutinho (2011, p.7) define las constantes como las características del sistema que no cambian en el periodo de interés. En este trabajo, las constantes están definidas por el peso relativo o ponderación que se asigna a cada competencia, la que depende de las características específicas del sector y las particularidades propias de la investigación. 
Para Anderson (2000), el mayor peso relativo fue dado a las competencias estratégicas y ello se debe a dos razones: la internacionalización es un asunto asociado a la estrategia y la visión de los gerentes dueños de las pymes, y a que esta fue la competencia que contó con un mayor número de preguntas (23 en total).

Es de resaltar que aunque la competencia de personal haya sido una de las de menor peso, debido al número de preguntas que la conformaron, no significa ello que no sea relevante, puesto que debe tenerse en cuenta que el presente trabajo se sustenta en los modelos de capital intelectual de Bueno y Morcillo (1997) y Sáez de Viteri (2000).

Para entender el porqué del peso relativo que se asignó a cada de las de las competencias citadas, es necesario conocer los ítems que las conforman. De esta manera se justifica el hecho de que las competencias estratégicas y tecnológicas tengan un mayor peso y las de personal y organizativo uno menor. Cabe recordar que dichos ítems sirvieron como base para la formulación de las preguntas que se utilizaron en el diseño del instrumento.

\section{Ítems del modelo de competencias básicas distintivas}

Ítems asociados a la competencia estratégica

Acerca de los ítems asociados a la competencia estratégica, Sáez de Viteri (2000) destaca los siguientes:

- Interés estratégico por permanecer en el sector

- Imagen y prestigio

- Localización e instalaciones

- Volumen de producción

- Cuota de mercado

- Rentabilidad

- Capacidad financiera

- Vulnerabilidad a los cambios en la demanda

- Barreras a la salida de la empresa

- Barreras a la movilidad dentro del grupo

Las preguntas realizadas en la encuesta tomaron como base cada uno de dichos ítems (Anexo A: Banco de preguntas DCBD). Por tratarse de una competencia de 
Diseño de un modelo de dirección por competencias básicas distintivas para las PYMES exportadoras de la ciudad de Medellín

tipo estratégico, la intención de cada una de las preguntas apuntaba hacia el conocimiento de la visión del empresario, su grado actual de inserción internacional, el compromiso de los recursos de la empresa con el propósito de internacionalización y la orientación de largo plazo de la misma.

\section{Ítems asociados a la competencia tecnológica}

En lo que respecta a la competencia tecnológica, los ítems que sirvieron como base para la elaboración de las preguntas fueron:

- Productos diferenciados y exclusivos

- Procesos de gran valor añadido

- Dominio de la tecnología

- Capacidad de innovación

- Activos estratégicos difíciles de imitar

- Flexibilidad productiva

- Sistemas de calidad

En este tipo de competencia las preguntas estuvieron orientadas hacia el conocimiento de las innovaciones en procesos y en productos de las organizaciones encuestadas (Anexo A: Banco de preguntas DCBD). La idea principal era, entonces, estar al tanto del stock o estado actual de la tecnología para conocer los recursos tecnológicos con los que cuenta cada empresa encuestada.

\section{Ítems asociados a la competencia de personal}

Por su parte, la competencia de personal tuvo como base para sus correspondientes preguntas los ítems que se enuncian a continuación:

- Aptitudes y formación

- Habilidades del personal

- Actitud y comportamiento personal

- Formación del capital humano

- Posición en la curva de experiencia

Con ello se quieren conocer los recursos de personal de los que disponen las organizaciones para cumplir el objetivo de internacionalización trazado en este trabajo. 


\section{Ítems asociados a las competencias organizativas}

Como se ha mencionado a lo largo del texto, buena parte de la ventaja competitiva de las organizaciones se encuentra sustentada en la forma como estas hacen las cosas. Por esta razón, los ítems a los que se encuentran asociadas las preguntas concernientes a dichas competencias son los siguientes:

- Dirección estratégica

- Capacidad directiva

- Diseño de tareas y métodos

- Estructura de poder

- Sistemas de información

Lo anterior se representa por medio de una matriz (Anexo B: Matriz de competencias básicas distintivas con orientación hacia la internacionalización), que pretende revelar cómo la estructura y la organización de la empresa hacen que fluya la información, los procesos, tareas y rutinas, y cómo ello se traduce en una ventaja competitiva y genera un potencial de internacionalización para las firmas encuestadas.

\section{Medidas y escalas de medición del modelo}

Para el presente trabajo se utilizaron escalas de medición de intervalo, propias de la estadística no probabilística. Las escalas de intervalo sirven para medir las diferencias absolutas entre todos los puntos de escala; estas diferencias son comparables. El punto de origen es arbitrario. En este caso particular, los resultados del instrumento reflejan los rangos que permiten conocer si la competencia esencial es valiosa, rara o exclusiva.

A continuación se describe el rango en el que serán ubicadas las empresas a encuestar, no sin aclarar primero que dicho intervalo está determinado por los resultados obtenidos en cada una de las competencias básicas distintivas, cuya sumatoria trae como resultado el puntaje obtenido total, el que debe ser comparado con el puntaje esperado que en este caso es de 160 que es el resultado de trabajar una matriz de 40 X 4. (Anexo B: Matriz de competencias básicas distintivas con orientación hacia la internacionalización).

Según Bueno y Morcillo (1997), para identificar y entender la competencia esencial hay, primero, que recordar que ella expresa la suma de las competencias básicas distintivas. En este trabajo el formato que se utilizó fue el de escala de Linkert 
Diseño de un modelo de dirección por competencias básicas distintivas para las PYMES exportadoras de la ciudad de Medellín

combinado con unas de diferencial semántico ${ }^{4}$. (Anexo B: Matriz de competencias básicas distintivas con orientación hacia la internacionalización).

Las preguntas fueron 40, todas daban la posibilidad de responder 4 opciones, lo que permite formar una matriz de 40 por 4 . Cada una de las opciones representa un mayor grado de complejidad de sus recursos, capacidades y rutinas, y su compromiso con la internacionalización. (Anexo B: Matriz de competencias básicas distintivas con orientación hacia la internacionalización)

La competencia esencial será valiosa cuando el puntaje se ubique en el rango (40-100). El puntaje de origen, es decir 40, es el valor obtenido si todas las respuestas de los empresarios fueran la opción 1, y el extremo superior, esto es, 100 se obtiene de un valor, considerado por los investigadores como media de una empresa con características normales, esto es, ni en un nivel superior ni en uno inferior. En este caso se tomó la situación en que 20 de las preguntas fueran resueltas mediante la opción 2 y las 20 restantes con la opción 3. Para este tipo de clasificación la organización tendrá unos recursos valiosos pero deberá cerrar todavía varias de sus brechas, lo que índica que se encontraría en una situación no apta para la internacionalización.

De la misma forma, la competencia esencial será considerada como rara si el puntaje obtenido en la encuesta se ubica en el rango 101-120. El extremo inferior representa la situación frontera entre la competencia esencial valiosa y la rara; el extremo superior representa el escenario en el que las respuestas seleccionadas equivalen, en promedio, a 10 para la opción 2, 20 para la opción 3 y 10 en la opción 4. Cuando una empresa posee una competencia esencial rara quiere decir que se encuentra en un nivel superior a la media. En este caso, la organización ya no será tan común ni tan corriente; al contrario, contaría con unos recursos avanzados pero que necesitan gestionarse mejor para que la firma se expanda de manera decidida en los mercados internacionales.

Por su parte, la competencia esencial será exclusiva si el puntaje obtenido se ubicara en el rango (121 - 160) ya que el límite inferior representa la situación límite o frontera entre la competencia esencial rara y la exclusiva; el límite superior expresa la situación en la que todas las respuestas asignadas fueran la opción 4, valga decir, la situación ideal. De esta forma, el puntaje obtenido será igual al puntaje esperado si y solo si todas las respuestas obtenidas fueran la opción 4. Como se sabe, este representaría un escenario muy poco probable puesto que no existe la organización perfecta.

$4 \quad$ Las escalas de Linkert indican el grado de acuerdo o desacuerdo que tienen con una serie de enunciados de creencias mentales o conductuales sobre un determinado objeto. Por su parte, las escalas con diferencial semántico captan las actitudes o sentimientos de la persona sobre un determinado objeto.

Semestre Económico, volumen 15, №. 32, pp. 197-224 • ISSN 0120-6346, julio-diciembre de 2012, Medellín, Colombia 
Es de destacar que para evitar la tendencia de muchos empresarios a responder las opciones 3 y 4, bien sea por temor de revelar las debilidades de su empresa o por una percepción demasiada optimista de la realidad, se realizaron algunas preguntas de control con el fin de validar la información registrada.

Adicional a ello, en el caso en que la competencia esencial fuera de carácter exclusivo se hizo una clasificación extra que consistió en valorarla como un activo imitable si el rango se ubicara entre 121-140. Su límite inferior, como ya se dijo, es el límite o frontera superior de la competencia rara, y su límite superior vendrá dado cuando en promedio 20 de las respuestas fueran la opción 3 y las otras 20 la situación 4.

Si el rango de respuestas se ubicase entre 141-160 se trata, entonces, de un activo inimitable. Para esta última clasificación, el límite inferior es el punto frontera con respecto a la competencia imitable, y el límite superior vendrá dado por la situación ideal, es decir, el caso en el que todas las preguntas sean respondidas con la opción 4.

Cuando una empresa se ubique en la categoría de exclusiva quiere decir que posee unas competencias superiores que las distancian de sus competidores. De esta forma impide que sus activos estratégicos sean copiados o reproducidos.

\section{HALLAZGOS}

El diseño metodológico acá desarrollado genera una mejora incremental con base en los modelos de competencias básicas distintivas abordados por Sáez de Viteri (2000) y Bueno y Morcillo (1997) debido a dos asuntos:

Primero, la cuantificación de las distintas competencias usa unas escalas de medición de Linkert combinadas con unas de diferencial semántico utilizadas para dar cuatro tipos de respuestas; ello no se encuentra en los modelos de Sáez de Viteri (2000) y Bueno y Morcillo (1997), es decir, ellos sirven como guía o como norte para formular las preguntas llevadas a la matriz de 40 por 40, pero las opciones de respuesta son elaboradas de una forma auténtica por quienes realizan este trabajo, de manera que al sumar los resultados se pudieran establecer los siguientes rangos de clasificación de la competencia esencial: valiosa entre 40- 100, rara entre 101-120 y exclusiva entre 121-160.

Segundo, en el modelo de Sáez de Viteri (2000) que tomó como referentes a Bueno y Morcillo (1997), los ítems que constituyen las competencias básicas distintivas son presentados de una forma genérica sin tener en cuenta si la empresa tiene su rango de actuación en la economía doméstica o en el ámbito internacional. En 
Diseño de un modelo de dirección por competencias básicas distintivas para las PYMES exportadoras de la ciudad de Medellín

el caso de este modelo se le ha dado un enfoque hacia la internacionalización; ello puede constatarse en que 9 de las 23 preguntas de la competencia estratégica se hicieron con una orientación hacia la internacionalización, lo que se constituye en un claro valor agregado que, por una parte, representa una innovación incremental en el estado del conocimiento de lo relacionado con las competencias básicas distintivas, y, por otra, las ubica dentro y solo dentro de las competencias estratégicas debido a que el tema de la internacionalización es, ante todo, un asunto estratégico.

El instrumento diseñado en este trabajo les permite a las empresas medir su potencial de internacionalización en términos de los recursos y capacidades que poseen, lo que se considera de mucha utilidad para aquellas empresas pymes que deseen iniciar un proceso de internacionalización, además de servir como herramienta de gestión para las mismas, pues permite identificar debilidades y fortalezas que tendrán que ser intervenidas si se quiere emprender operaciones más allá de las fronteras nacionales.

Este tipo de temática ayuda a comprender por qué la internacionalización se encuentra asociada a la visión del empresario, a los recursos que posee la empresa y a las capacidades que esta logre desarrollar. Lo importante de este tipo de modelos es que contribuyen a conocer la posición competitiva de las empresas, asunto este que se convierte en el primer paso para pensar en un proceso de internacionalización porque les permite a las organizaciones saber cuáles son los recursos y las capacidades con los que cuenta para internacionalizarse.

Este tipo de instrumentos lo pueden aplicar las empresas sin excepción ya que en su gran mayoría las competencias son genéricas; además, el tema de la internacionalización en el mundo de hoy es de importancia para casi todas las empresas, por lo que puede ser de gran utilidad, en primera instancia, para medir su potencial de internacionalización.

\section{CONCLUSIONES}

En el modelo de competencias básicas distintivas acá presentado, el puntaje obtenido por las empresas puede variar debido a dos factores: el primero es la forma como se hagan las preguntas y su orientación hacia la internacionalización; el segundo tiene que ver con las opciones de respuesta y su grado de profundidad. Sin embargo, el rigor con el que se ha diseñado el modelo permite que este sea aplicado como una alternativa para conocer el nivel de competencia esencial en el que se encuentra cada organización. 
El modelo presenta varios avances metodológicos y temáticos que permiten generar un aporte incremental e innovador a los modelos de competencias básicas distintivas. Lo metodológico tiene que ver con la orientación de las preguntas y las escalas de medición sobre las que se cimentaron las opciones de respuesta. Lo temático consiste en el hecho de vincular a las competencias básicas distintivas la orientación hacia la internacionalización.

Este modelo puede ser aplicado por todo tipo de sectores y empresas que deseen internacionalizarse, ya que las competencias son de tipo genérico. Lo que se espera para futuros trabajos es que esta herramienta se utilice con fines de representatividad estadística que sirvan para conocer características concretas de diferentes sectores.

La posición competitiva de las empresas estará definida por la competencia esencial; a medida que estas avanzan hacia una de tipo exclusivo significará que se encuentran más preparadas en términos de sus recursos y capacidades para la internacionalización. Por lo tanto este instrumento es de utilidad para medir el potencial de internacionalización de las empresas de tipo pyme.

\section{BIBLIOGRAFÍA}

AECA, (1999): La Dirección y Gestión por Competencias. En línea: Principios de Organización y Sistemas. Madrid, AECA, www.aeca.es/pub/documentos/pol1.htm. (15 de agosto de 2010).

Alcaldía de Medellín. (2008). Plan de Desarrollo. Medellín es solidaria y competitiva. Medellín. En linea: Alcaldía de Medellín, Medellín, agosto de 2008, http://aprendeenlinea.udea.edu. co/Ims/investigacion/file.php/39/ARCHIVOS 2010/PDF/Plan de Desarrollo Municipal articulado al PNDE.pdf. (10 de septiembre de 2010).

Amit, R. y Schoemaker, P. (1993). Strategic assets and organizational rent. En: Strategic Management Journal. Vol 14, N. ${ }^{\circ}$ 1, pp. 33-46.

Arango, M. D., Pérez, G. y Gil, H. (2008). Propuestas de modelos de gestión de capital intelectual: Una revisión. En: Contaduría Universidad de Antioquia, N. ${ }^{\circ}$ 52, pp. 105-130.

Atkinson, A; Waterhouse, J y Wells, R. B. (1997): A stakeholder approach to strategic performance measurement. En: Sloan Management Review, Spring, pp. 25-37.

Barney, J. (1991). Firm Resources and Susteined Competitive Advantage. En: Journal of Management. Vol 17, N. 1 , pp. 99-120.

Barney, J. (1986). Strategic factor markets expectations, luck and business strategy. En: Management Science, Vol 10, N. 32 , pp. 1.231-1.241. 
Diseño de un modelo de dirección por competencias básicas distintivas para las PYMES exportadoras de la ciudad de Medellín

Bontis, N. (1998): Intellectual Capital: an exploratory study that develops measures and models. En: Management Decision, Vol. 36, N. 2 2, pp. 63-76.

Bontis, N., Crossan, M. y Hulland, J. (2002). Managing an organizational learning systems by aligning stocks and flows. En: Journal of Management Studies, Vol 39, N. ${ }^{\circ}$, pp. 437-469.

Brooking, A. (1996): Intellectual Capital. Core Asset for the Third Millennium enterprise, International Thomson Business press, London. Vol 8, N. ${ }^{\circ}$ 12-13; p. 76.

Bueno, E.; Salmador, M. Y Merino, C (2008). Génesis, concepto y desarrollo del capital intelectual en la economía del conocimiento: una reflexión sobre el Modelo Intellectus y sus aplicaciones. En: Estudios de Economía Aplicada, Vol. 26, N. ${ }^{\circ}$ 2, pp. 43-64.

Bueno, E., \& Morcillo, P. (1997). Dirección por competencias básicas distintivas: Propuesta de un modelo de competitividad integral y evidencia empirica. Documento IADE, N. ${ }^{\circ}$ 51, pp. 1-39.

Calderón H. (2006) Competencias distintivas en las pyme: un aporte desde gestión humana. En: Innovar. Vol. 16, N. ${ }^{\circ} 27$, pp. 57-72.

Camisón, C. Palacios, D .y Devece, C. (2000): Un nuevo modelo para la medición del capital intelectual: el modelo Nova. En línea: ACEDE, Valencia, 7 de mayo de 2007, http:// riunet.upv.es/bitstream/handle/10251/1836/tesisUPV2605.pdf.txt;jsessionid=6ACD54 AAD23CC9505D837FB447F3BF98? sequence=21. (12 de septiembre de 2010).

Cardona J. A. Calderón, G. (2006). El impacto del aprendizaje en el rendimiento organizacional. En: Cuadernos de Administración, Vol. 19, N. 32, pp. 11-44.

Carrión, J. (2007). Estrategia: de la visión a la acción. Madrid, Esic Editorial. 462p.

Castells, Manuel. (1995). La ciudad informacional. Tecnologías de la Información, reestructuración económica y el proceso urbano-regional. Madrid: Alianza Editorial. 504p.

Crossan, M., Lane, H. y White, R. (1999). An organizational learning framework: From intuition to institution. En: Academy of Management Review, Vol. 24, N. 3, 522-537.

De Castro Díez y Jiménez Martín. (1995). El Comportamiento directivo como competencia distintiva. En: Investigaciones Europeas de Dirección y Economía de la Empresa Vol. 1, N. ${ }^{\circ} 2,1995$, pp. 87-98.

Dodgson, M. (1991): Technology, learning, technology strategy and competitive pressures. En: British Journal of Management, Vol. 2, N. ${ }^{\circ}$ 3, pp. 132-149.

Dodgson, M. (1993). Organizational learning: a review of some literatures. En: Organization Studies, Vol. 14, N. ${ }^{\circ}$ 3, pp. 375-395.

Edvinson, L. (1997): Developing intellectual capital at Skandia. En: Long Range Planning, Vol. 30, N. ${ }^{\circ}$ 3, pp. 366-373.

Semestre Económico, volumen 15, №. 32, pp. 197-224 • ISSN 0120-6346, julio-diciembre de 2012, Medellín, Colombia 
Fiol, M. y Lyles, M. (1985). Organizational learning. En: Academy of Management Review, Vol. 10, N. ${ }^{\circ} 4$, pp. 803-813.

Garvin, D. (1994). Building a learning organization. En: Business Credit. Vol. 96, N. 1, pp. 19-29.

Huber, G. (1999). Synergies between organizational learning and creativity and innovation. En: Creativity and Innovation Management, vol. 7, N. ${ }^{\circ}$ 1, pp. 3-8.

Huselid, M. (1995). The impact of human resource management practices on turnover, productivity and corporate financial performance. En: Academy of Management Journal. Vol. 1, N. ${ }^{\circ} 38$, pp. 635-672.

Lado, A. A. y Wilson, M. C. (1994). Human resource systems and sustained competitive advantage: toward a conceptual integration. En: Journal of management. Vol. 19, N. 4 , pp. 699-727.

Malaver, F. y Vargas, M. (2004). Los procesos de innovación en América Latina: aportes para su caracterización. En: Cuadernos de Administración. Vol. 17, N. 28, pp. 9-51.

Mcdougall, P \& Oviatt, B. (2000). International Entrepreneurship: The Intersection of Two Research Paths. En: Academy of Management Journal. Vol. 43, N. 5, pp. 902-906.

McElroy, M.W. (2001): Social innovation capital draft, Macroinnovation Associates, July, pp. $1-14$.

Morcillo, P. \& Bueno, E. (1996). Fundamentos de Economía y Organización Industrial. Madrid: McGraw Hill.

Morcillo, P., Rodríguez Antón, J. M., Casani, F. y Rodríguez Pomeda, J. (2000). El valor de los conocimientos y del aprendizaje como fuente de competencias básicas distintivas. En: Dirección y Organización, N. 24. Octubre, pp. 12-20.

Moutinho, L. (2011). Methodology and Methods. Foundation Chair of Marketing. University of Glasgow. pp. 1-27.

Moutinho, L. (2011). Seminario Internacional de Metodología de la Investigación. Escuela de Ingenierías de Antioquia.

Navas, J., E Guerra, L. (1998). La Dirección Estrategica de la Empresa. Teorías y Aplicaciones. Madrid: Civitas.

Nelson, R. (1995). Recent evolutionary theorizing about economic change. En: Journal of Economic. Vol. XXXIII, pp. 48-90.

Nelson, R; Winter, S., (1982). An evolutionary theory of economic change. Harvard University Press. Cambridge. Massachusetts. 439 p.

Penrose, E. (1959). The teory of the growth of the firm. New York: Oxford University Press. 
Diseño de un modelo de dirección por competencias básicas distintivas para las PYMES exportadoras de la ciudad de Medellín

Pfeffer, J. (1996). Ventaja competitiva a través de la gente: cómo desencadenar el poder de la fuerza de trabajo. México: CECSA

Pickett, S. (2000). Developing internal audit competencies. En: Managerial Auditing Journal. Vol. 15, N. ${ }^{\circ}$ 6, pp. 265-278.

Prahalad, C., \& Hamel, G. (1990). The Core Competence Of The Corporation. En: Harvard Business Review. Vol. 68, N. 3, pp. 79-91.

Pulakos, E.D:, Schmitt, N. y Chan, D. (1996) Models of job performance rating: an examination of rate race, rate gender and rater level effects. En: Human Performance. Vol. 9, N. ${ }^{\circ}$, pp. 103-119.

Quinn, J; Hilmer, F. (1994). Strategic outsourcing. En: Sloan Management Review. Vol 35, N. 4 ; pp. 43-55.

Sakaiya, T. (1985). The Knowledge Value Revolution or a History of the Future. Kodansha America, $379 \mathrm{p}$.

Saez de Viteri (2000). El potencial competitivo de la empresa: recursos, capacidades, rutinas y procesos de valor añadido. En: Investigaciones Europeas de Dirección. Vol. 6, N. 3 , pp. $71-86$.

Selznick, P. (1957). Leadership in Administration. NY: Haper \& Row, 162 p.

Skandia Report (1994): Visualizing Intellectual Capital in Skandia, Skandia Insurance Company Ltd, Estocolmo, 259 p.

Simon, H (1957). A Behavioral Model of Rational Choice. En Models of Man, Social and Rational: Mathematical Essays on Rational Human Behavior in a Social Setting. New York: Wiley.

Slater, S. y Narver, J. (1995). Market orientation and the learning organization. Journal of Marketing, Vol. 59, N. ${ }^{\circ}$, pp. 63-75.

Sveiby, K.E. (1997): The New Organizational Wealth: Managing and measuring Knowledge-based assets, Berrett-Kochler, San Francisco, 275 p.

Tsang, E. (1997). Organizational learning and the lerning organization: a dichotomy between descriptive and prescriptive research. En: Human Relation. Vol. 50, N. ${ }^{\circ}$ 3, pp. 73-89.

Ulrich, E., E. Wiersema, M. (1989). Gaining Strategic and Organizational Capability in a Turbulent. En: The Academy of Management Executive. Vol. 3, N. ${ }^{\circ}$ 2, pp. 115-122.

Uribe Gonzalez, E. M. (2001). Operaciones Internacionales. En: Cuadernos de Administración. Vol. 14, N. 23 , pp. 25-49.

Wernefelt, B (1984). A resource based view of the firm". En: Strategic management Journal, Vol. 5, N. ${ }^{\circ}$ 2, pp. $171-180$.

Semestre Económico, volumen 15, №. 32, pp. 197-224 • ISSN 0120-6346, julio-diciembre de 2012, Medellín, Colombia 